\title{
Interrupted Time-Series for the Evaluation of Affordable Care Act Impact on Stereotactic Radiosurgery for the Treatment of Brain Metastases
}

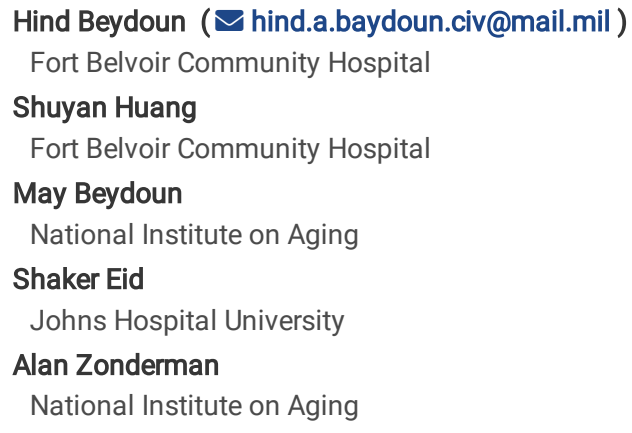

Research Article

Keywords: Brain, Hospitalization, Metastasis, Radiation, Stereotactic.

Posted Date: January 25th, 2021

DOI: https://doi.org/10.21203/rs.3.rs-147005/v1

License: (a) (1) This work is licensed under a Creative Commons Attribution 4.0 International License. Read Full License 


\section{Abstract}

Background: The 2010 Affordable Care Act aimed at reducing healthcare costs, improving healthcare quality and expanding health insurance coverage among uninsured individuals in the United States. We examined trends in utilization of radiation therapies and stereotactic radiosurgery before and after its implementation among U.S. adults hospitalized with brain metastasis.

Methods: Interrupted time-series analyses of data on 383934 2005-2014 Nationwide Inpatient Sample hospitalizations were performed, whereby yearly and quarterly cross-sectional data were evaluated and Affordable Care Act implementation was considered the main exposure variable, stratifying by patient and hospital characteristics.

Results: We observed consistently declining trends in radiation therapy over time and post-Affordable Care Act status with variability in level of utilization among specific sub-groups. Stereotactic radiosurgery prevalence increased over time among Hispanics, elective admissions, Midwestern hospitals, nonteaching hospitals and hospitals with medium bed size. Post-Affordable Care Act was associated with increased stereotactic radiosurgery prevalence among African-Americans, non-elective and weekend admissions, with changes in slope in the context of weekend admissions and hospitals with large bed size.

Conclusions: Whereas hospitalized adults in the United States utilized less radiation therapy and slightly more stereotactic radiosurgery over the ten-year period, utilization levels and trends were not consistent among distinct sub-groups defined by patient and hospital characteristics, with some traditionally underserved populations more likely to receive healthcare services post-Affordable Care Act implementation. The Affordable Care Act may be helpful at reducing the need for radiation therapy and closing the gap in access to technological advances such as stereotactic radiosurgery for treating brain metastases.

\section{Background}

With nearly 200,000 incident cases per year, brain metastases are the most frequently diagnosed intracranial cancers among adults in the United States. Treatment of brain metastases frequently involves radiation therapy(1), as the standard treatment has been whole brain radiation therapy (WBRT) with or without surgical resection(2). When treated with supportive care and corticosteroids (median survival: 1-2 months) or WBRT (median survival: 3-6 months), brain metastases are known to exhibit poor prognosis(3). Available since the 1980s, stereotactic radiosurgery (SRS) is a minimally invasive procedure that is considered an alternative to WBRT that can quickly deliver ablative doses of radiation while minimizing radiation exposure to normal brain tissue and risks of cognitive side-effects and/or neurologic compromise(1-5). SRS may offer a one-day treatment course with prolonged treatment planning while avoiding or delaying side-effects; by contrast, WBRT is a less expensive option that reduces intracranial relapse and allows faster initiation of radiation treatment(2).

Previously conducted studies have provided evidence for increasing popularity of SRS among cancer patients, in general, and among those with brain metastases, in particular $(3,6,7)$. Guadognolo and colleagues analyzed claims data from Surveillance, Epidemiology, and End Results (SEER)-Medicare and Texas Cancer Registry-Medicare (2000-2009) databases on utilization of radiation therapy among 13488 patients diagnosed with lung, breast, prostate, colorectal, melanoma, and pancreas cancers within their last 30 days of life(7). Results suggested a shift towards technologically advanced treatments, with a decrease in utilization of two-dimensional radiation therapy $(74.9 \%(2000)$ to $32.7 \%(2009))$, and an increase in utilization of three-dimensional radiation therapy (27.2\%(2000) to $58.5 \%(2009)$, intensity modulated radiation therapy (0\%(2000) to 6.2\%(2009)) and SRS (0\%(2000) to 5.0\%(2009))(7). Halasz and colleagues performed analyses of SEER-Medicare data on 7684 elderly patients with non-small cell lung cancer (NSCLC) diagnosed with brain metastases and treated initially with radiation therapy within two months of their diagnosis between 2000 and 2007(2). Whereas 469 (6.1\%) patients had billing codes for SRS, SRS utilization increased from 3.0\% (2000) to 8.2\% (2005) and varied by registry site. Predictors of SRS utilization included increasing year of diagnosis, SEER registry, higher socioeconomic status, admission at teaching hospital, no history of participation in low-income state buy-in programs, no extracranial metastases, and longer interval from NSCLC diagnosis(2). Haque and colleagues analyzed data on 2,312 patients (813 SRS and 1,499 non-SRS) from 2005-2014 National Cancer Database (NCD) to evaluate utilization of intracranial radiotherapy for renal cell carcinoma; SRS utilization increased from $27 \%$ in 2005 to $44 \%$ in 2014 , and was more frequent among patients whose place of residence was away from the facility, those who were treated at academic centers and/or had chemotherapy and/or nephrectomy $(P<0.05)$. SRS was less frequent among those with lower income and who were uninsured/had Medicaid(1).

Recently, trends in SRS and non-SRS radiation therapies for brain metastases were likely influenced by the 2010 Affordable Care Act (ACA) which was signed into law to reduce healthcare costs, improve healthcare quality and expand health insurance coverage among uninsured individuals in the United States(821). The ACA had several provisions facilitating access to cancer-related procedures among previously underserved populations(8-21). To date, studies focused on the impact of the ACA on utilization of procedures aimed at prevention, diagnosis and treatment of cancers have been scarce and have yielded inconsistent findings(8-21). Moreover, studies examining time trends in SRS utilization for treating brain metastases among U.S. adults have mainly originated from SEER-Medicare and NCD and none specifically examined the potential role of ACA using an all-payer database at the national level. The main objective of this retrospective study is to perform interrupted time-series (ITS) analyses to examine variations in time trends pertaining to utilization of radiation therapies, in general, and SRS, in particular, among hospitalized U.S. adults diagnosed with brain metastasis over a 10-year period between 2005 and 2014. We hypothesized that levels and trends in utilization differed before and after ACA implementation and, that patient and hospital-level characteristics modified the difference and/or change in utilization pre- and post-implementation of the ACA.

\section{Methods}

We performed secondary analyses of existing data from the Agency for Healthcare Research and Quality (AHRQ), Healthcare Cost and Utilization Project (HCUP), Nationwide Inpatient Sample (NIS). The AHRQ HCUP NIS is the largest publicly available, all-payer inpatient care database of community hospitals 
in the United States. It consists of $\approx 5-8$ million hospital discharge records sampled annually from $\approx 1000$ hospitals since 1988 . Each year, a $20 \%$ stratified probability sample of hospitals (before 2012) or hospital discharge records (since 2012) is selected from all participating HCUP states. NIS data elements included patient demographics, up to 15 diagnoses and 15 procedures as well as hospital course and outcomes. This study was conducted in accordance with the Declaration of Helsinki and was determined to be research not involving human subjects by Fort Belvoir Community Hospital.

The study population consists of hospital discharge records from 2005-2014 NIS databases that met inclusion criteria: (1) Age $\geq 18$ years; (2) Primary or secondary diagnosis of brain metastases based on ICD-9-CM codes (191 (malignant neoplasm brain), 191.7 (malignant neo brain stem), 191.8 (malignant neo brain nec), 191.9 (malignant neo brain nos), 198.3 (sec mal neo brain/spine)). Hospital discharge records were excluded if they corresponded to patients with missing data on patient- and hospital-level characteristics.

Eligible discharge records were identified as corresponding to patients receiving radiation therapy if $1+$ of up to 15 ICD-9-CM procedure codes were the following: SRS (92.3(stereotact radiosurgery*), 92.30(stereo radiosurgery nos), 92.39 (stereo radiosurgery nec)) or non-SRS (92.2(therap radiol \& nucl med*), 92.21(superficial radiation), 92.22(orthovoltage radiation), 92.23(radioisot teleradiother), 92.24(teleradio using photons), 92.25(electron teleradiotherap), 92.26(particul teleradioth nec), 92.27(radioactive elem implant), 92.29(radiotherapeut proc nec), 92.31 (sing source radiosurgery), 92.32(multisource radiosurgery), 92.33(particulate radiosurgery)). Records that did not satisfy these criteria were identified as corresponding to patients who did not receive SRS and/or non-SRS therapies during their hospitalization. Subsequently, prevalence of radiation therapy among hospitalized patients was defined as proportion of eligible records that corresponded to patients who underwent SRS and/or non-SRS treatments and prevalence of SRS was defined as proportion of eligible radiation therapy records that corresponded to patients who received SRS.

Prevalence rates were examined over a 10-year study period (2005-2014) as well as before (2005-2010) and after (2011-2014) ACA implementation. Twenty-four pre-intervention (before quarter 1 of 2011) and sixteen post-intervention (since quarter 1 of 2011) time points were generated using year and quarter of hospital discharge, and time trends were examined before and after stratifying by patient and hospital characteristics. Patient-level characteristics were sex, age, race/ethnicity, Charlson comorbidity index (CCI), admission type, admission quarter, weekend admission and primary health insurance. Hospital-level characteristics were hospital region, location/teaching status and bed size.

All statistical analyses were conducted using STATA version 15 (StataCorp, College Station, TX), taking into account complex survey design. Bivariate associations were examined using uncorrected Chi-square and design-based F-tests, as appropriate. First, logistic regression models were constructed for comparing distributions of patient and hospital characteristics among hospitalizations that occurred pre-post ACA implementation. Second, prevalence rates of radiation therapies and SRS were evaluated at each point in time, and trend analyses were performed by year and quarter using logistic regression. We used time point-specific prevalence rates to perform comparative ITS analyses by examining differences in levels and slopes over time between periods prepost ACA implementation, before and after stratifying by patient or hospital characteristics(20). ITS analysis is a robust quasi-experimental method for evaluating the impact of population-level interventions through estimation of change in outcome around the time of an intervention while avoiding individual-level unmeasured confounding and controlling for pre-existing time trends prior to that intervention(10, 15, 20). All ITS models included indicator variables for time point (year and quarter coded as $51, \ldots, 144)$, post-intervention status and an interaction term between time point and post-intervention status(18). We applied post-estimation to calculate change in intercept and slope for pre-post ACA prevalence rates(11, 21), and calculated Newey-West standard errors to adjust for autocorrelation $(10,15,20)$. We also constructed Poisson regression models whereby each patient and hospital characteristic, post-ACA status and their interactions were examined as predictors of prevalent radiation therapy and SRS. Statistical tests were two-sided and $\mathrm{p}<0.05$ was considered statistically significant.

\section{Results}

Of 388174 hospitalizations identified among U.S. adults with primary or secondary diagnosis of brain metastases from 2005-2014 NIS databases, 383934 were study-eligible because they had no missing data on baseline characteristics. Of those, 228567 were pre-ACA and 155367 were post-ACA records. Similarly, 35928 underwent any type of radiation therapy with 2833 undergoing SRS and 34406 undergoing non-SRS therapies (Fig. 1).

Table 1 compares patient and hospital characteristics of eligible records pre-post ACA implementation. Whereas pre-ACA and post-ACA records did not differ by patient sex or admission quarter, we observed small but statistically significant differences for all remaining patient and hospital characteristics.

Compared to pre-ACA records, post-ACA records were more likely to correspond to older, minority patients with $\mathrm{CCl}>2$ admitted on weekends to Midwestern, Southern or Western urban-teaching hospitals, and less likely to correspond to non-Medicare patients electively admitted to urban, non-teaching hospitals with medium or large bed sizes. 
Table 1

Characteristics of Brain Metastasis Patients Before and After the Affordable Care Act

\begin{tabular}{|c|c|c|c|c|c|}
\hline & Weighted \% & & & cOR $(95 \% \mathrm{Cl})$ & aOR $(95 \% \mathrm{Cl})$ \\
\hline & Total & Pre-ACA & Post-ACA & & \\
\hline & $(n=383,934)$ & 2005-2010 & 2011-2014 & & \\
\hline & & $(n=228,567)$ & $(n=155,367)$ & & \\
\hline Sex: & & $P=0.056$ & & & \\
\hline Male & 48.9 & 49.1 & 48.7 & Ref. & Ref. \\
\hline Female & 51.0 & 50.9 & 51.3 & $1.01(0.99,1.02)$ & $1.01(0.99,1.02)$ \\
\hline Age (years): & & $P<0.0001$ & & & \\
\hline $18-39$ & 6.9 & 7.1 & 6.7 & Ref. & Ref. \\
\hline $40-64$ & 50.5 & 51.1 & 49.8 & $1.03(1.01,1.06)$ & $1.06(1.04,1.09)$ \\
\hline $65+$ & 42.4 & 41.8 & 43.4 & $1.11(1.07,1.14)$ & $1.04(1.01,1.08)$ \\
\hline Race/Ethnicity: & & $P<0.0001$ & & & \\
\hline White & 65.3 & 61.5 & 70.9 & Ref. & Ref. \\
\hline African American & 9.6 & 8.5 & 11.1 & $1.14(1.11,1.16)$ & $1.03(1.00,1.05)$ \\
\hline Hispanic & 5.6 & 5.2 & 6.4 & $1.06(1.03,1.09)$ & $1.05(1.02,1.08)$ \\
\hline Other & 4.7 & 4.2 & 5.4 & $1.09(1.06,1.013)$ & $1.09(1.05,1.12)$ \\
\hline Unknown & 14.8 & 20.6 & 6.2 & $0.26(0.25,0.26)$ & $0.21(0.20,0.22)$ \\
\hline Charlson comorbidity index: & & $P<0.0001$ & & & \\
\hline$\leq 2$ & 14.4 & 14.8 & 13.8 & Ref. & Ref. \\
\hline $3-9$ & 83.3 & 83.3 & 83.3 & $1.06(1.04,1.08)$ & $1.02(1.00,1.04)$ \\
\hline$\geq 10$ & 2.3 & 1.9 & 2.9 & $1.58(1.52,1.66)$ & $1.41(1.34,1.48)$ \\
\hline Admission type: & & $P<0.0001$ & & & \\
\hline Non-Elective & 79.9 & 78.9 & 81.4 & Ref. & Ref. \\
\hline Elective & 20.0 & 21.1 & 18.6 & $0.85(0.84,0.87)$ & $0.86(0.85,0.88)$ \\
\hline Admission quarter: & & $P=0.18$ & & & \\
\hline 1st quarter & 24.9 & 25.0 & 24.9 & Ref. & Ref. \\
\hline 2nd quarter & 24.9 & 24.9 & 24.8 & $1.00(0.98,1.02)$ & $0.99(0.98,1.01)$ \\
\hline 3rd quarter & 25.0 & 24.9 & 25.1 & $1.01(0.99,1.03)$ & $1.01(0.99,1.03)$ \\
\hline 4th quarter & 25.1 & 25.0 & 25.3 & $1.02(0.99,1.03)$ & $1.01(0.99,1.03)$ \\
\hline Weekend admission status: & & $P<0.0001$ & & & \\
\hline Monday-Friday & 80.7 & 81.0 & 80.2 & Ref. & Ref. \\
\hline Saturday-Sunday & 19.3 & 18.9 & 19.8 & $1.06(1.04,1.07)$ & $1.04(1.02,1.06)$ \\
\hline Primary health insurance: & & $P<0.0001$ & & & \\
\hline Medicare & 44.2 & 42.9 & 46.0 & Ref. & Ref. \\
\hline Medicaid & 11.8 & 11.3 & 12.5 & $1.02(1.00,1.05)$ & $0.96(0.93,0.98)$ \\
\hline Private insurance & 37.9 & 39.6 & 35.4 & $0.84(0.82,0.85)$ & $0.83(0.81,0.84)$ \\
\hline Self-pay/No pay/Other & 6.2 & 6.2 & 6.2 & $0.93(0.91,0.96)$ & $0.88(0.86,0.91)$ \\
\hline Hospital region: & & $P<0.0001$ & & & \\
\hline Northeast & 21.9 & 22.6 & 20.9 & Ref. & Ref. \\
\hline Midwest & 23.2 & 23.4 & 22.9 & $1.06(1.04,1.07)$ & $1.77(1.75,1.79)$ \\
\hline South & 36.9 & 36.1 & 37.9 & $1.13(1.12,1.14)$ & $1.37(1.35,1.38)$ \\
\hline West & 17.9 & 17.9 & 18.0 & $1.08(1.07,1.09)$ & $1.32(1.30,1.34)$ \\
\hline
\end{tabular}




\begin{tabular}{|c|c|c|c|c|c|}
\hline & \multicolumn{3}{|c|}{ Weighted \% } & \multirow[t]{2}{*}{$\operatorname{cOR}(95 \% \mathrm{Cl})$} & \multirow[t]{2}{*}{ aOR (95\% Cl) } \\
\hline \multicolumn{2}{|c|}{ Hospital location/teaching status: } & \multicolumn{2}{|c|}{$P<0.0001$} & & \\
\hline Rural & 8.3 & 9.1 & 7.1 & Ref. & Ref. \\
\hline Urban -Non-Teaching & 32.3 & 35.2 & 28.1 & $1.01(0.99,1.03)$ & $0.93(0.92,0.95)$ \\
\hline Urban - Teaching & 59.4 & 55.7 & 64.8 & $1.48(1.46,1.51)$ & $1.48(1.46,1.52)$ \\
\hline \multicolumn{2}{|l|}{ Hospital bed size: } & \multicolumn{2}{|c|}{$P<0.0001$} & & \\
\hline Small & 10.2 & 9.8 & 10.7 & Ref. & Ref. \\
\hline Medium & 20.9 & 20.3 & 21.9 & $0.99(0.98,1.01)$ & $0.96(0.94,0.98)$ \\
\hline Large & 68.9 & 69.9 & 67.4 & $0.88(0.88,0.89)$ & $0.89(0.88,0.90)$ \\
\hline
\end{tabular}

Abbreviations: $\mathrm{ACA}=$ Affordable Care Act; $\mathrm{Cl}=$ Confidence Intervals; OR=Odds Ratio.

Table 2 presents trends in utilization of radiation therapy and SRS. In general, $9.34 \%$ of brain metastasis patients underwent radiation therapies and $7.83 \%$ of brain metastasis patients who underwent radiation therapies underwent SRS. There were statistically significant trends by year and/or quarter in radiation therapy and SRS prevalence rates. Whereas trend in radiation therapy declined over time, that of SRS is less clear-cut.

Table 2

Time Trends in Utilization of Radiation Therapies and Stereotactic Radiosurgery for Brain Metastasis

\begin{tabular}{|c|c|c|c|c|c|c|c|c|}
\hline & \multicolumn{4}{|c|}{ \% Radiation Therapy ${ }^{a}$} & \multicolumn{4}{|c|}{ \% Stereotactic Radiosurgery ${ }^{b}$} \\
\hline & Quarter 1 & Quarter 2 & Quarter 3 & Quarter 4 & Quarter 1 & Quarter 2 & Quarter 3 & Quarter 4 \\
\hline 2005 & 11.71 & 11.94 & 11.10 & 11.0 & 7.41 & 6.65 & 4.95 & 7.08 \\
\hline 2006 & 12.16 & 11.51 & 11.43 & 10.90 & 9.44 & 9.28 & 7.88 & 6.21 \\
\hline 2007 & 11.12 & 11.18 & 10.49 & 10.61 & 7.87 & 7.51 & 7.67 & 7.32 \\
\hline 2008 & 10.42 & 9.83 & 9.62 & 9.76 & 9.04 & 8.59 & 6.61 & 7.20 \\
\hline 2009 & 10.32 & 9.25 & 9.39 & 8.80 & 7.52 & 6.32 & 6.12 & 7.71 \\
\hline 2010 & 9.05 & 8.80 & 8.89 & 8.51 & 9.47 & 8.60 & 9.35 & 5.83 \\
\hline 2011 & 8.80 & 9.09 & 8.59 & 8.58 & 9.99 & 8.17 & 7.36 & 7.75 \\
\hline 2012 & 8.58 & 8.38 & 8.09 & 8.04 & 8.15 & 6.61 & 7.53 & 7.51 \\
\hline 2013 & 8.14 & 7.61 & 7.67 & 7.52 & 7.51 & 7.64 & 8.80 & 9.48 \\
\hline 2014 & 7.27 & 7.07 & 6.77 & 6.73 & 8.50 & 9.70 & 8.47 & 10.52 \\
\hline Total & 9.34 & & & & 7.83 & & & \\
\hline$P_{\text {trend }}($ Year) & $<0.0001$ & & & & 0.013 & & & \\
\hline$P_{\text {trend }}$ (quarters) & $<0.0001$ & & & & 0.008 & & & \\
\hline$P_{\text {trend }}$ (Year \& quarters) & $<0.0001$ & & & & 0.002 & & & \\
\hline
\end{tabular}

Table 3 presents ITS and Poisson regression analyses for ACA as a predictor of prevalent radiation therapy and SRS. Overall, prevalence of radiation therapy declined over time, with significant increase in prevalence (change in intercept) around the time of ACA implementation, and no significant change in slope post-ACA (Fig. 2). There was no significant change in intercept or slope with respect to SRS prevalence over time, suggesting no significant effect of ACA on SRS prevalence among radiation therapy recipients (Fig. 3). Poisson regression models suggested a negative relationship between post-ACA status and radiation therapy (Incidence Rate Ratio $(\mathrm{IRR})=0.77,95 \%$ Confidence Interval $(\mathrm{CI}): 0.76,0.78$ ) and a weak but positive relationship between post-ACA status and SRS (IRR $=1.09,95 \% \mathrm{Cl}: 1.02,1.18)$.

Table 3. Interrupted Time Series and Poisson Regression for Radiation Therapy and Stereotactic Radiosurgery Utilization 


\begin{tabular}{|c|c|c|c|c|c|c|c|c|}
\hline & \multicolumn{4}{|c|}{ Radiation Therapy ${ }^{a}$} & \multicolumn{4}{|c|}{ Stereotactic Radiosurgery ${ }^{b}$} \\
\hline & \multicolumn{3}{|l|}{ ITSA } & \multirow[t]{2}{*}{ PR } & \multicolumn{3}{|l|}{ ITSA } & PR \\
\hline & \multicolumn{3}{|l|}{$\beta(95 \% \mathrm{Cl})$} & & \multicolumn{4}{|l|}{$\beta(95 \% \mathrm{Cl})$} \\
\hline & $-t$ & - x111 & X_t111 & $\begin{array}{l}\text { IRR }(95 \% \\
\mathrm{Cl}) \\
\mathrm{P}_{\text {interact }}\end{array}$ & $-t$ & - 1111 & _x_t111 & $\begin{array}{l}\text { IRR (95\% Cl) } \\
P_{\text {interact }}\end{array}$ \\
\hline \multirow[t]{2}{*}{ Total } & -0.0005876 & \multirow{2}{*}{$\begin{array}{l}0.0057787 \\
(0.0021209 \\
0.0094366)\end{array}$} & -0.0000186 & \multirow{2}{*}{$\begin{array}{l}0.77 \\
(0.76 \\
0.78)\end{array}$} & 0.0001294 & -0.0028808 & 0.0002599 & \multirow{2}{*}{$\begin{array}{l}10.09 \\
1.18)\end{array}$} \\
\hline & $\begin{array}{l}(-0.0006893 \\
-0.0004858)\end{array}$ & & $\begin{array}{l}(-0.0001516 \\
0.0001144)\end{array}$ & & $\begin{array}{l}(-0.0002191 \\
0.0004779)\end{array}$ & $\begin{array}{l}(-0.0199838 \\
0.0142222)\end{array}$ & $\begin{array}{l}(-0.0003722 \\
0.000892)\end{array}$ & \\
\hline \multicolumn{9}{|l|}{ Sex: } \\
\hline \multirow[t]{3}{*}{ Male } & \multirow{3}{*}{$\begin{array}{l}-0.0005697 \\
(-0.0006869 \\
-0.0004526)\end{array}$} & 0.0045067 & 0.0000369 & \multirow{2}{*}{$\begin{array}{l}0.77 \\
(0.75 \\
0.79)\end{array}$} & \multirow{3}{*}{$\begin{array}{l}0.0001567 \\
(-0.0002992 \\
0.0006127)\end{array}$} & \multirow{3}{*}{$\begin{array}{l}-0.0024153 \\
(-0.0244049 \\
0.0195743)\end{array}$} & 0.0001599 & $10.09(0.98,1.21)$ \\
\hline & & $\begin{array}{l}(-0.0001133 \\
0.0091267)\end{array}$ & $\begin{array}{l}(-0.0001345 \\
0.0002083)\end{array}$ & & & & $\begin{array}{l}(-0.0004606 \\
0.0007804)\end{array}$ & Ref. \\
\hline & & & & Ref. & & & & \\
\hline Female & -0.0006019 & 0.0068577 & -0.0000727 & 0.77 & 0.0000993 & -0.0029964 & 0.0003499 & $10.09(0.99,1.22)$ \\
\hline & $\begin{array}{l}(-0.000 / 24 \\
-0.0004799)\end{array}$ & $\begin{array}{l}(0.0014591 \\
0.0122562)\end{array}$ & $(-0.0002573$ & $\begin{array}{l}(0.75 \\
0.79)\end{array}$ & $(-0.0003332$ & $(-0.02334$ & $(-0.0004782$ & 0.950 \\
\hline & & & & 0.804 & & & & \\
\hline Age (years). & & & & & & & & \\
\hline $18-39$ & -0.0004489 & 0.0121922 & -0.0001827 & 0.81 & 0.0005132 & -0.0280496 & 0.0011782 & $1.14(0.89,1.46)$ \\
\hline & $(-0.000723$ & $0.023624)$ & $(-0.0007689$ & $0.88)^{\prime}$ & $(-0.0006543$, & $(-0.0947041$ & $(-0.001746$ & Ref. \\
\hline & $-0.0001748)$ & & & Ref. & & & & \\
\hline $40-64$ & -0.0004611 & 0.0017223 & -0.0000759 & 0.79 & 0.0002121 & -0.0072218 & -0.0000104 & $10.04(0.94,1.15)$ \\
\hline & $(-0.0005616$ & $(-0.0029789$, & $(-0.0002382$ & $0.81)^{\prime}$ & $(-0.0002433$ & $(-0.0313832$ & $(-0.0009374$ & 0.482 \\
\hline & $-0.0003605)$ & & & 0.680 & $0.0000070)$ & (1) & & \\
\hline $65+$ & -0.0007671 & 0.0095095 & 0.0000941 & 0.74 & -0.0000161 & 0.0048715 & 0.0005076 & $1.17(10.04,1.32)$ \\
\hline & $(-0.0009341$ & $0.0154606)$ & $(-0.0001515$ & $0.77)$ & $(-0.0003611$ & $(-0.0150786$ & $(-0.0003607$ & 0.847 \\
\hline & $-0.0006001)$ & & & 0.136 & & & & \\
\hline Race/Ethnic & & & & & & & & \\
\hline White & -0.000553 & 0.0049148 & -0.0000157 & 0.77 & 0.0000306 & -0.0044736 & 0.0004462 & 10.04 \\
\hline & $(-0.0006734$ & $(-0.0001796$ & $(-0.0001946$ & $0.79)^{\prime}$ & $(-0.0004324$ & $(-0.0265235$ & $(-0.0002873$ & 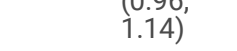 \\
\hline & $-0.0004326)$ & & & Ref. & & & & Ref. \\
\hline African & -0.00063 & 0.0080009 & -0.0002553 & 0.80 & -0.0001931 & 0.0253202 & 0.0003061 & 1.52 \\
\hline & $(-0.0010712$ & $(-0.0091001)$ & $(-0.000812$ & $0.85)$ & $0.0003174)$ & $0.0448371)$ & $(-0.000464$ & $1.99)^{\prime}$ \\
\hline & $-0.0001888)$ & & & 0.291 & & & & 00.008 \\
\hline Hispanic & -0.0002661 & -0.0178086 & -0.0002545 & 0.66 & 0.0013095 & 0.006235 & -0.0033457 & 1.22 \\
\hline & $\begin{array}{l}(-0.0007323 \\
0.0002001)\end{array}$ & $-0.0018471)$ & $\begin{array}{l}(-0.0008252 \\
0.0003163)\end{array}$ & $0.72)^{\prime}$ & $0.0022193)^{\prime}$ & $\begin{array}{l}(-0.0638439 \\
0.0763139)\end{array}$ & $(-0.0062731$ & 1.57) \\
\hline & & & & 00.001 & & & $-00.0004184)$ & 0.282 \\
\hline Other & -0.0010128 & 0.0120473 & 0.0004902 & 0.73 & 0.0003647 & -0.0030949 & -0.000435 & $\begin{array}{l}1.14 \\
081\end{array}$ \\
\hline & $(-0.0016214$ & $(-0.0068166$ & $(-0.000326$ & $0.80)^{\prime}$ & $(-0.0006091$ & $(-0.0538316$ & $(-0.0027238$ & $1.61)^{\prime}$ \\
\hline & $-0.0004041)$ & & & 0.256 & & & & 0.607 \\
\hline Unknown & - & - & - & $\begin{array}{l}0.79 \\
(0.74 \\
0.85)\end{array}$ & - & - & - & $\begin{array}{l}10.06 \\
(0.83 \\
1.37)\end{array}$ \\
\hline & & & & 0.555 & & & & 0.889 \\
\hline
\end{tabular}




\begin{tabular}{|c|c|c|c|c|c|c|c|c|}
\hline $\begin{array}{l}\text { Charlson } \\
\text { comorbidity } \\
\text { index: }\end{array}$ & & & & & & & & \\
\hline$\leq 2$ & -0.0003402 & 0.0042873 & 0.0001067 & 0.70 & 0.0001474 & -0.082355 & 0.0046514 & 0.98 \\
\hline & $(-0.000469$ & $(-0.0013229$ & $(-0.0001005$ & $0.78)$ & $(-0.0017997$ & $(-.1924038$ & $(-0.0002966$ & $1.14)^{\prime}$ \\
\hline & $-0.0002114)$ & 0.00989/5) & 0.000314) & & $0.0020945)$ & $0.0216938)$ & U.0095994) & \\
\hline $3-9$ & -0.0006299 & 0.0045373 & -0.0000306 & 0.76 & 0.0002095 & 0.0000498 & -0.0000586 & 1.14 \\
\hline & $(-0.0007463$ & $0.008999)$ & $(-0.0002008$ & $0.77)$ & $(-0.0000873$ & $(-0.0153025$ & $(-0.0006743$ & $\begin{array}{l}\text { (10.U5, } \\
1.24)\end{array}$ \\
\hline & $-0.0005135)$ & & & 0.124 & & & & 00.080 \\
\hline$\geq 10$ & -0.0014818 & 0.0615762 & -0.0000477 & $\begin{array}{l}0.95 \\
0.85\end{array}$ & -0.000659 & 0.0214923 & 0.0026537 & 2.17 \\
\hline & $(-0.002198$ & $0.089895)$ & $(-0.0009786$ & $10.07)$ & $(-0.001567$ & $(-0.0201997$, & $0.0046317)^{\prime}$ & $3.99)^{\prime}$ \\
\hline & $-0.0007655)$ & & & $<00.0001$ & & & & 00.013 \\
\hline $\begin{array}{l}\text { Type of } \\
\text { admission: }\end{array}$ & & & & & & & & \\
\hline Elective & & & 0.0001221 & $\begin{array}{l}0.79 \\
0.77\end{array}$ & $\begin{array}{l}0.0018591 \\
0.0005552\end{array}$ & -.1039817 & 0.0000293 & $\begin{array}{l}0.94 \\
(085\end{array}$ \\
\hline & $(-0.0009787$ & $\begin{array}{l}(-0.0072935, \\
0.0140144)\end{array}$ & $\begin{array}{l}(-0.0002302) \\
0.0004743)\end{array}$ & $0.80)$ & $0.003163)$ & $(-.1762625$ & $(-0.0036463$ & $10.06)$ \\
\hline & $-0.0004928)$ & & & Ref. & & $-0.0317009)$ & & Ref. \\
\hline Non-Elective & -0.0005618 & 0.0065739 & -0.000071 & 0.65 & -0.0000744 & 0.0158522 & 0.0004508 & $\begin{array}{l}1.42 \\
(129\end{array}$ \\
\hline & $(-0.0006493$ & $0.0105927)$ & $\begin{array}{l}(-0002319 \\
0.0000899)\end{array}$ & $0.69)$ & $\begin{array}{l}(-0.0003229 \\
0.0001741)\end{array}$ & $0.0263995)$ & $0.0007904)$ & $1.56)$ \\
\hline & $-0.0004743)$ & & & $<00.0001$ & & & & $<00.0001$ \\
\hline $\begin{array}{l}\text { Admission } \\
\text { quarter. }\end{array}$ & & & & & & & & \\
\hline $1^{\text {st }}$ quarter & - & - & - & $\begin{array}{l}0.76 \\
0.73\end{array}$ & - & - & - & $10.02(0.88,1.17)$ \\
\hline & & & & $0.79)$ & & & & Ref. \\
\hline & & & & Ref. & & & & \\
\hline $2^{\text {nd }}$ quarter & - & - & - & $\begin{array}{l}0.77 \\
(0.74\end{array}$ & - & - & - & $10.02(0.88,1.18)$ \\
\hline & & & & $0.80)^{\prime}$ & & & & 0.971 \\
\hline & & & & 0.558 & & & & \\
\hline $3^{\text {rd }}$ quarter & - & - & - & $\begin{array}{l}0.77 \\
0.74\end{array}$ & - & - & - & $1.13(0.97,1.31)$ \\
\hline & & & & $0.80)$ & & & & 0.321 \\
\hline & & & & 0.754 & & & & \\
\hline $4^{\text {th }}$ quarter & - & - & - & $\begin{array}{l}0.78 \\
0.75\end{array}$ & - & - & - & $1.26(10.08,1.46)$ \\
\hline & & & & $0.81)$ & & & & 00.037 \\
\hline & & & & 0.446 & & & & \\
\hline $\begin{array}{l}\text { Weekend } \\
\text { admission } \\
\text { status: }\end{array}$ & & & & & & & & \\
\hline Monday- & -0.0006352 & 0.005331 & 0.0000585 & 0.76 & 0.000209 & -0.009793 & 0.0002213 & $10.04(0.96,1.13)$ \\
\hline & $\begin{array}{l}(-0.0007601 \\
-0.0005102)\end{array}$ & $0.0095893)$ & $\begin{array}{l}(-0.0000884 \\
0.0002054)\end{array}$ & $0.78)$ & $\begin{array}{l}(-0.0001878 \\
0.0006059)\end{array}$ & $\begin{array}{l}(-0.0316774, \\
0.0120915)\end{array}$ & $\begin{array}{l}(-0.000604 \\
0.0010465)\end{array}$ & Ref. \\
\hline & & & & Ref. & & & & \\
\hline Saturday- & -0.0003808 & 0.0074733 & -0.0003339 & 0.82 & -0.0001375 & 0.0288493 & 0.0002812 & $1.60(1.30,1.96)$ \\
\hline & $\begin{array}{l}(-0.0004951 \\
-0.0002666)\end{array}$ & $\begin{array}{l}(-0.0027994, \\
0.017746)\end{array}$ & $\begin{array}{l}(-0.0007438, \\
0.000076)\end{array}$ & $0.86)^{\prime}$ & $\begin{array}{l}(-0.0004831 \\
0.0002081)\end{array}$ & $0.0456)$ & $\begin{array}{l}(-0.0006933, \\
0.0012558)\end{array}$ & $<00.0001$ \\
\hline & & & & 00.006 & & & & \\
\hline $\begin{array}{l}\text { Primary health } \\
\text { insurance: }\end{array}$ & & & & & & & & \\
\hline
\end{tabular}




\begin{tabular}{|c|c|c|c|c|c|c|c|c|}
\hline Medicare & $\begin{array}{l}-0.0008394 \\
(-0.0010039 \\
-0.000675)\end{array}$ & $\begin{array}{l}0.0114842 \\
(0.0055055, \\
0.0174629)\end{array}$ & $\begin{array}{l}0.0001623 \\
(-0.0000891 \\
0.0004138)\end{array}$ & $\begin{array}{l}0.74 \\
(0.72 \\
0.76) \\
\text { Ref. }\end{array}$ & $\begin{array}{l}-0.0000486 \\
(-0.0003595 \\
0.0002622)\end{array}$ & $\begin{array}{l}0.0002031 \\
(-0.0172791 \\
0.0176854)\end{array}$ & $\begin{array}{l}0.0007014 \\
(-0.0000317 \\
0.0014345)\end{array}$ & $\begin{array}{l}1.12(10.00,1.26) \\
\text { Ref. }\end{array}$ \\
\hline \multirow[t]{2}{*}{ Medicaid } & -0.0003131 & 0.0050678 & -0.000596 & \multirow{2}{*}{$\begin{array}{l}0.83 \\
(0.78 \\
0.87) \\
<00.0001\end{array}$} & -0.0002999 & 0.0259187 & 0.00037 & $1.30(10.06,1.60)$ \\
\hline & $\begin{array}{l}(-0.0004865 \\
-0.0001396)\end{array}$ & $\begin{array}{l}(-0.0037625 \\
0.013898)\end{array}$ & $\begin{array}{l}(-0.0009537 \\
-0.0002382)\end{array}$ & & $\begin{array}{l}(-0.001019 \\
0.0004192)\end{array}$ & $\begin{array}{l}(-0.0099386 \\
0.061776)\end{array}$ & $\begin{array}{l}(-0.001016 \\
0.001756)\end{array}$ & 0.221 \\
\hline \multirow{2}{*}{$\begin{array}{l}\text { Private } \\
\text { insurance }\end{array}$} & -0.0003918 & -0.002866 & 0.0000137 & \multirow{2}{*}{$\begin{array}{l}0.77 \\
(0.75 \\
0.80) \\
00.068\end{array}$} & 0.0004478 & -0.0154042 & -0.0001583 & $10.04(0.93,1.17)$ \\
\hline & $\begin{array}{l}(-0.0004903 \\
-0.0002933)\end{array}$ & $\begin{array}{l}(-0.0083102 \\
0.0025782)\end{array}$ & $\begin{array}{l}(-0.0001948 \\
0.0002223)\end{array}$ & & $\begin{array}{l}(-0.0001163 \\
0.001012)\end{array}$ & $\begin{array}{l}(-0.0429707 \\
0.0121624)\end{array}$ & $\begin{array}{l}(-0.001129 \\
0.0008124)\end{array}$ & 0.386 \\
\hline \multirow{2}{*}{$\begin{array}{l}\text { Self-pay/No } \\
\text { pay/Other }\end{array}$} & -0.0006988 & \multirow{2}{*}{$\begin{array}{l}0.020173 \\
(0.0047719 \\
0.035574)\end{array}$} & -0.0002168 & \multirow{2}{*}{$\begin{array}{l}0.82 \\
(0.76 \\
0.89)\end{array}$} & 0.0003893 & 0.0016599 & -0.0004993 & $1.18(0.86,1.64)$ \\
\hline & $\begin{array}{l}(-0.0010599 \\
-0.0003377)\end{array}$ & & $\begin{array}{l}(-0.0007284 \\
0.0002947)\end{array}$ & & $\begin{array}{l}(-0.00051 \\
0.0012886)\end{array}$ & $\begin{array}{l}(-0.0511885 \\
0.0545083)\end{array}$ & $\begin{array}{l}(-0.0024409 \\
0.0014423)\end{array}$ & 0.741 \\
\hline
\end{tabular}




\begin{tabular}{|c|c|c|c|c|c|c|c|c|}
\hline \multicolumn{9}{|l|}{ Hospital region: } \\
\hline \multirow[t]{3}{*}{ Northeast } & -0.000183 & -0.0115514 & -0.0003083 & \multirow{2}{*}{$\begin{array}{l}0.79 \\
(0.76 \\
0.82)\end{array}$} & -0.0001349 & -0.0270346 & 0.0011506 & \multirow{2}{*}{$\begin{array}{l}0.82 \\
(0.70 \\
0.95)\end{array}$} \\
\hline & $(-0.000384$ & $(-0.0231198$ & $(-0.0007614$ & & \multirow{2}{*}{$\begin{array}{l}(-0.001105 \\
0.0008352)\end{array}$} & \multirow{2}{*}{$\begin{array}{l}(-0.0682845 \\
0.0142153)\end{array}$} & \multirow{2}{*}{$\begin{array}{l}(-0.0002096 \\
0.0025108)\end{array}$} & \\
\hline & & & & Ref. & & & & Ref. \\
\hline \multirow[t]{3}{*}{ Midwest } & -0.0006922 & \multirow{3}{*}{$\begin{array}{l}0.0153239 \\
(0.0080416, \\
0.0226063)\end{array}$} & 0.0000413 & \multirow{2}{*}{$\begin{array}{l}0.82 \\
(0.78 \\
0.86)\end{array}$} & \multirow{3}{*}{$\begin{array}{l}0.0009955 \\
(0.0004157 \\
0.0015753)\end{array}$} & -0.0245319 & -0.0006917 & \multirow{2}{*}{$\begin{array}{l}1.24 \\
(10.05 \\
1.45)\end{array}$} \\
\hline & $(-0.0009179$ & & $(-0.0002486$, & & & $(-0.0510817$ & $(-0.0016173$ & \\
\hline & $-0.0004664)$ & & & 0.206 & & & & $<00.0001$ \\
\hline \multirow[t]{3}{*}{ South } & -0.0005641 & 0.0046105 & -0.0001032 & \multirow{2}{*}{$\begin{array}{l}0.76 \\
(0.74 \\
0.79)\end{array}$} & 0.0001402 & 0.0076084 & 0.0004799 & \multirow{2}{*}{$\begin{array}{l}1.32 \\
(1.17 \\
1.48)\end{array}$} \\
\hline & $(-0.0007274$ & $(-0.002617$ & $(-0.0003507$ & & \multirow{2}{*}{$\begin{array}{l}(-0.0003511 \\
0.0006316)\end{array}$} & \multirow{2}{*}{$\begin{array}{l}(-0.017319 \\
0.0325357)\end{array}$} & \multirow{2}{*}{$\begin{array}{l}(-0.0004077 \\
0.0013676)\end{array}$} & \\
\hline & $-0.0004008)$ & 0.01 & $0.0001445)$ & 0.159 & & & & $<00.0001$ \\
\hline \multirow[t]{3}{*}{ West } & -0.0009943 & \multirow{3}{*}{$\begin{array}{l}0.0189184 \\
(0.0102924, \\
0.0275444)\end{array}$} & \multirow{3}{*}{$\begin{array}{l}0.0004228 \\
(0.000105, \\
0.0007406)\end{array}$} & \multirow{2}{*}{$\begin{array}{l}0.69 \\
(0.66 \\
0.74)\end{array}$} & -0.0007519 & 0.0463469 & -0.0003994 & \multirow{2}{*}{$\begin{array}{l}10.00 \\
(0.84 \\
1.20)\end{array}$} \\
\hline & & & & & \multirow[t]{2}{*}{$\begin{array}{l}(-0.0018053 \\
0.0003014)\end{array}$} & \multirow[t]{2}{*}{$\begin{array}{l}(-0.0038763 \\
0.09657)\end{array}$} & \multirow{2}{*}{$\begin{array}{l}(-0.0023635 \\
0.0015648)\end{array}$} & \\
\hline & $-0.0007399)$ & & & 00.001 & & & & 00.092 \\
\hline \multicolumn{9}{|c|}{$\begin{array}{l}\text { Hospital } \\
\text { location/teaching } \\
\text { status: }\end{array}$} \\
\hline Rural & 0.0003967 & -0.0274169 & -0.0006766 & 0.69 & 0.0011802 & -0.0623366 & -0.0009277 & 10.00 \\
\hline & $0.0006446)$ & $(-0.0405148$ & $(-0.0010602$ & $0.74)^{\prime}$ & $0.0019127)^{\prime}$ & $(-0.1084894$ & $(-0.0022133$ & $1.20)^{\prime}$ \\
\hline & & $-0.0143191)$ & $-0.000293)$ & Ref. & & $-0.0161837)$ & & Ref. \\
\hline Urban -Non- & -0.0007415 & 0.0162992 & -0.0002277 & 0.73 & 0.0006459 & -0.0067011 & -0.0006939 & 0.52 \\
\hline & $(-0.0008881$ & $0.023398)$ & $\begin{array}{l}(-0.0004992 \\
0.0000437)\end{array}$ & $0.81)$ & $0.0011458)$ & $\begin{array}{l}(-0.0359347 \\
0.0225325)\end{array}$ & $\begin{array}{l}(-0.0019489 \\
0.0005611)\end{array}$ & $0.90)$ \\
\hline & $-0.000595)$ & & & 0.848 & & & & 00.001 \\
\hline Urban - & -0.000724 & 0.0057822 & 0.0000952 & 0.74 & -0.0003339 & 0.0074095 & 0.000628 & 1.37 \\
\hline & $(-0.0008464$ & $(-0.000209$ & $(-0.0001343$ & $0.77)^{\prime}$ & $(-0.0008084$ & $(-0.0124293$, & $(-0.0001396$ & $1.63)^{\prime}$ \\
\hline & $-0.0006017)$ & $0.0171784)$ & $0.0003<40)$ & 0.566 & 0.00 & 0.0 & (200700 & 00.021 \\
\hline Hospital bed si & & & & & & & & \\
\hline Small & -0.0006084 & 0.0063257 & 0.0001727 & 0.75 & -0.0006939 & 0.0324438 & -0.0006291 & 0.80 \\
\hline & $(-0.000833$ & $(-0.0043283$ & $(-0.0001905$ & $0.80)^{\prime}$ & $(-0.0030342$, & $(-0.028774$ & $(-0.00338$ & $10.02)$ \\
\hline & $-0.0003839)$ & & & Ref. & & & & Ref. \\
\hline Medium & -0.0005753 & 0.003114 & 0.0001367 & 0.74 & 0.0007184 & -0.0054802 & -0.0015508 & 10.08 \\
\hline & $(-0.0008217$ & $(-0.0066312$ & $(-0.0002968$ & $0.78)$ & $0.0013808)$ & $(-0.0556636$, & $(-0.0034093$ & $1.28)$ \\
\hline & $-0.0003289)$ & & & 0.819 & & & & 00.049 \\
\hline Large & -0.000584 & 0.0056636 & -0.0000206 & 0.79 & 0.0001169 & -0.0064868 & 0.000772 & 1.14 \\
\hline & $(-0.0006839$ & $0.0104542)$ & $(-0.0001839$ & $0.81)^{\prime}$ & $(-0.0003076$ & $(-0.024428$ & $0.0013598)$ & $1.24)$ \\
\hline & $-0.0004841)$ & & & 0.146 & & & & 00.006 \\
\hline
\end{tabular}

a Of all hospitalizations corresponding to patients who were diagnosed with brain metastases; b Of all hospitalizations corresponding to patients who were diagnosed with brain metastases and underwent radiation therapies; Abbreviations: IRR = Incidence Rate Ratio; ITSA = Interrupted Time Series Analysis; PR = Poisson Regression; _t = Time point effect; _x111 = Post-ACA effect; _ $\mathrm{x} \_t 111$ = Post-ACA by time effect.

We observed ITS disparities after stratifying by each patient and hospital characteristic. Although declining trend in radiation therapy was consistently observed in all sub-groups, post-ACA status only impacted patients who were female, 18-39 or $\geq 65$ years of age, those with CCl $>2$, non-elective admissions, weekday admissions, Medicare, self-pay, no pay or other insurance, and those admitted to Midwestern, Western urban, non-teaching or large hospitals, with a negative effect of post-ACA status on rural hospitals. Stratified ITS analyses for radiation therapy showed no statistically significant time by post-ACA status interaction effects, suggesting trends were similar pre-post ACA. ITS analyses of SRS showed that SRS prevalence increased over time among Hispanics and in the context of elective admissions, Midwestern hospitals, non-teaching hospitals and hospitals with medium bed size. Furthermore, we observed a positive impact of ACA on SRS utilization among African-Americans, non-elective and weekend admissions, with significant change in slope among weekend admissions and hospitals with large bed size. Similar disparities by patient and hospital characteristics were observed in the context of stratified Poisson regression models. 


\section{Discussion}

In this series of cross-sectional studies, we used multiple time points defined based on year and quarter of hospital admission from NIS (2005-2014) and ITS analyses to examine whether ACA had an impact on time trends in utilization of radiation therapies and SRS among hospitalized U.S. adult patients diagnosed with brain metastases. Key findings were as follows: (1) There was a decline in radiation therapy utilization with no change in rate of decline prepost ACA; (2) Post-ACA, radiation therapy utilization shifted downwards for rural hospitals and upwards for certain patient (females, 18-39 or $\geq 65$ years of age, $\mathrm{CCl}>2$, non-elective admissions, weekday admissions, Medicare, self-pay, no pay or other insurance) and hospital (Midwestern or Western urban-nonteaching and large hospitals) sub-groups; (3) There was no clear trend in SRS utilization, except for Hispanics, elective admissions, Midwestern, non-teaching and medium bed-size hospitals; (4) Post-ACA, African-Americans, non-elective and weekend admissions experienced upward shift in SRS utilization; (5) PostACA, weekend admissions and large bed-size hospitals experienced increasing SRS rates.

Previously conducted ITS analyses focused on the potential role of ACA on utilization of healthcare services have examined specific ACA provisions, including Medicaid expansion( $9,10,12,13,16,21)$, elimination of cost-sharing expenses for preventive services $(8,17,18,20)$ and overall ACA implementation $(8,11,15,17,19,20)$. Given the complexity of this issue, few studies specifically focused on cancer diagnosis, prevention and treatment prepost ACA and these studies yielded inconsistent findings, with evidence for improved healthcare access among underserved populations(8, 10, 11, 14, 15, 17-21). For instance, Carlos and colleagues used patient-level analytic files on 1763959 commercially insured women aged 40 to 74 years from the $2004-$ 2014 Clinformatics Data Mart to evaluate changes in mammography cost sharing and utilization pre-post ACA, while comparing these outcomes by age group, and found limited impact of ACA on cost-sharing and utilization due to simultaneous revision of USPSTF guidelines(11). By contrast, Haakenstaad performed ITS analyses using 2009-2012 data on commercially-insured and Medicare beneficiaries 50-75 years from the Maine Health Data Organization All-Payer Claims Database to estimate ACA impact on trends in rural-urban disparities in colonoscopy rates and costs, and found decline in urban-rural disparities that was attributed to ACA implementation(14). Using data on 44343 insured individuals, 50-64 years, who participated in $2007-2015$ Medical Expenditure Panel Survey, Mbah and colleagues performed ITS to estimate the impact of ACA cost-sharing provision on ethnic disparities in colorectal cancer screening and found increased utilization that was not significantly different between Hispanics with non-Hispanic patients (17). An ITS analysis was conducted by Steenland and colleagues using Massachusetts All-Payer Claims Database (2009-2012) to evaluate changes in cost and utilization trends for breast, cervical and colorectal cancer screenings post-ACA implementation(20). Whereas ACA was associated with decline in weekly copayment for preventive breast and cervical cancer screenings, the likelihood of copayment for colon cancer screening declined throughout the study period, with decline rate slowing after ACA(20). There was, however, only weak evidence for ACA impact on increased rates of cancer screening(20). Lu and colleagues performed ITS analyses using JPS Center for Cancer Care institutional registry data on 4808 urban, underserved, adult patients diagnosed with a first primary invasive solid tumor between 2008 and 2015, to examine the impact of ACA implementation on stage at diagnosis in Texas, a Medicaid nonexpansion state(15). Their results suggested that ACA implementation decreased the prevalence of uninsured cancer patients but had little effect on cancer stage at diagnosis(15). Moss and colleagues examined the impact of the ACA Medicaid expansion (2008-2010 vs. 2011-2014 and 2014 vs. 2011-2013) on insurance rates among 181866 women diagnosed with cervical, uterine or ovarian cancer using 2008-2014 SEER data(19). The study found a significant increase in Medicaid enrollment after 2011, and a significant decrease in uninsured rates for all cancer types between 2011 and 2014(19). After January 2014 , uninsured rates decreased by $50 \%$ for uterine and ovarian cancer and by $25 \%$ for cervical cancer(19). These changes were noted only among U.S. States that expanded Medicaid coverage(19).

To our knowledge, this study is the first to examine the potential role of ACA implementation on utilization of radiation therapy and SRS using an all-payer database at the national level. Study findings should, nevertheless, be interpreted with caution and in light of several limitations. First, we relied on an administrative database consisting of discharge records with limited scope and granularity. Particularly, hospital location was identified as U.S. region rather than U.S. State, precluding our ability to stratify discharge records according to implementation of the ACA Medicaid expansion as well as our ability to compare the time before and after its implementation. Furthermore, unlike the SEER-Medicare and NCD databases, the HCUP NIS database does not collect detailed information on cancer diagnosis, staging and treatment. Second, complete subject analysis was performed with potential for selection bias because of missing data. Third, many study variables, including brain cancer diagnosis and treatment, were defined using ICD-9 codes, potentially leading to misclassification bias. Fourth, although ITS may be considered as a quasi-experimental method, residual confounding may have led to biased differences in outcome among distinct sub-groups. Fifth, the role of chance cannot be eliminated given the limited number of patients who underwent radiation therapy and SRS. Sixth, the cross-sectional design of each wave of NIS does not allow establishment of temporality or causal relationships between variables of interest. Finally, study results can only be generalized to hospitalized patients within the period of interest, whose characteristics may differ from those who sought outpatient care for SRS and/or non-SRS radiation therapies at later time points.

\section{Conclusions}

In conclusion, hospitalized U.S. adults with brain metastases utilized less radiation therapy and slightly more SRS over time between 2005 and 2014. However, utilization levels and trends were not consistent among distinct sub-groups defined by patient and hospital characteristics, with some traditionally underserved populations more likely to receive healthcare services post-ACA implementation. These results are consistent with some of the previously published studies and with the idea that the ACA may be helpful at reducing the need for radiation therapy as well as closing the gap in access to technologically advanced treatments for brain metastases such as SRS. Delays in cancer diagnosis and treatment may be responsible for health disparities whereby racial and ethnic minorities are more likely to present at later cancer stages to the healthcare system and to experience higher cancer-related morbidity and mortality rates as compared to whites. Future research are needed to explore the question of whether the ACA and its specific provisions can promote earlier diagnosis and treatment of brain metastases, thus reducing morbidity and mortality risks among minorities and other underserved populations. 


\title{
List Of Abbreviations
}

\author{
$\mathrm{ACA}=$ Affordable Care Act \\ AHRQ = Agency for Healthcare Research and Quality \\ $\mathrm{CCl}=$ Charlson comorbidity index \\ HCUP $=$ Healthcare Cost and Utilization Project \\ ITS = interrupted time-series \\ $\mathrm{NCD}=$ National Cancer Database \\ NIS = Nationwide Inpatient Sample \\ NSCLC $=$ non-small cell lung cancer \\ SEER = Surveillance, Epidemiology, and End Results \\ SRS $=$ stereotactic radiosurgery \\ WBRT = whole brain radiation therapy \\ IRR = Incidence Rate Ratio
}

\section{Declarations}

Ethics approval and consent to participate: This manuscript is based on a project that received the determination of research not involving human subjects. Accordingly, the need for ethics approval and consent to participate was waived by Fort Belvoir Community Hospital Institutional Review Board. Administrative permissions and/or licenses were acquired by our research team through a Data Use Agreement with the Agency for Healthcare Research and Quality to access the patient data used in this research.

Consent for publication: Not applicable.

Availability of data and materials: The data that support the findings of this study are available from the Agency for Healthcare Research and Quality but restrictions apply to the availability of these data, which were used under license for the current study, and so are not publicly available. Data are however available from the authors upon reasonable request and with permission of Agency for Healthcare Research and Quality.

Competing interests: The authors declare that they have no competing interests.

\section{Funding: None.}

Authors' contributions: HB conceived the research study, performed data management and statistical analyses and drafted the manuscript. SH performed literature reviews and revised the manuscript for intellectual content. MB participated in statistical analysis and revised the manuscript for intellectual content. SE provided access to the data, participated in statistical analysis and revised the manuscript for intellectual content. AZ oversaw the research study and revised the manuscript for intellectual content. All authors read and approved the final manuscript.

Acknowledgements: The manuscript is supported in part by the Intramural Research Program of the National Institute on Aging in Baltimore, Maryland.

Disclaimer: The views expressed in this article are those of the authors and do not reflect the official policy of Fort Belvoir Community Hospital, the Defense Health Agency, Department of Defense, or the U.S. Government. Any discussion or mention of commercial products or brand names does not imply or support any endorsement by the Federal Government.

\section{References}

1. Haque W, Verma V, Butler EB, Teh BS. Utilization of Stereotactic Radiosurgery for Renal Cell Carcinoma Brain Metastases. Clinical genitourinary cancer. 2018.

2. Halasz LM, Weeks JC, Neville BA, Taback N, Punglia RS. Use of stereotactic radiosurgery for brain metastases from non-small cell lung cancer in the United States. International journal of radiation oncology, biology, physics. 2013;85(2):e109-16.

3. Rydzewski NR, Khan AJ, Strauss JB, Chmura SJ. Mortality After Stereotactic Radiosurgery for Brain Metastases and Implications for Optimal Utilization: A National Cancer Database Study. American journal of clinical oncology. 2018.

4. Avbovbo UE, Appel SJ. Strategies to Alleviate Anxiety Before the Placement of a Stereotactic Radiosurgery Frame. The Journal of neuroscience nursing: journal of the American Association of Neuroscience Nurses. 2016;48(4):224-8.

5. Miller JA, Balagamwala EH, Angelov L, Suh JH, Rini B, Garcia JA, et al. Spine stereotactic radiosurgery with concurrent tyrosine kinase inhibitors for metastatic renal cell carcinoma. Journal of neurosurgery Spine. 2016;25(6):766-74. 
6. Fetcko K, Lukas RV, Watson GA, Zhang L, Dey M. Survival and complications of stereotactic radiosurgery: A systematic review of stereotactic radiosurgery for newly diagnosed and recurrent high-grade gliomas. Medicine. 2017;96(43):e8293.

7. Guadagnolo BA, Liao KP, Giordano SH, Elting LS, Buchholz TA, Shih YC. Increasing use of advanced radiation therapy technologies in the last 30 days of life among patients dying as a result of cancer in the United States. Journal of oncology practice. 2014;10(4):e269-76.

8. Agenor M, Murchison GR, Chen JT, Bowen DJ, Rosenthal MB, Haneuse S, et al. Impact of the Affordable Care Act on human papillomavirus vaccination initiation among lesbian, bisexual, and heterosexual U.S. women. Health services research. 2020;55(1):18-25.

9. Aliu O, Giladi AM, Chung KC. Trends in Medicaid beneficiaries' receipt of breast reconstruction procedures following Pre-Affordable Care Act (ACA) Medicaid expansion in New York State. American journal of surgery. 2018;216(3):551-7.

10. Al-Refaie WB, Zheng C, Jindal M, Clements ML, Toye P, Johnson LB, et al. Did Pre-Affordable Care Act Medicaid Expansion Increase Access to Surgical Cancer Care? Journal of the American College of Surgeons. 2017;224(4):662-9.

11. Carlos RC, Fendrick AM, Kolenic G, Kamdar N, Kobernik E, Bell S, et al. Breast Screening Utilization and Cost Sharing Among Employed Insured Women After the Affordable Care Act. Journal of the American College of Radiology: JACR. 2019;16(6):788-96.

12. Crocker AB, Zeymo A, Chan K, Xiao D, Johnson LB, Shara N, et al. The Affordable Care Act's Medicaid expansion and utilization of discretionary vs. nondiscretionary inpatient surgery. Surgery. 2018;164(6):1156-61.

13. Giladi AM, Aliu O, Chung KC. The effect of medicaid expansion in new york state on use of subspecialty surgical procedures by medicaid beneficiaries and the uninsured. Journal of the American College of Surgeons. 2014;218(5):889-97.

14. Haakenstad A, Hawkins SS, Pace LE, Cohen J. Rural-urban disparities in colonoscopies after the elimination of patient cost-sharing by the Affordable Care Act. Preventive medicine. 2019;129:105877.

15. Lu Y, Jackson BE, Gehr AW, Cross D, Neerukonda L, Tanna B, et al. Affordable Care Act and cancer stage at diagnosis in an underserved population. Preventive medicine. 2019;126:105748.

16. Mahendraratnam N, Dusetzina SB, Farley JF. Prescription Drug Utilization and Reimbursement Increased Following State Medicaid Expansion in 2014. Journal of managed care \& specialty pharmacy. 2017;23(3):355-63.

17. Mbah OM, Kinlaw AC, Trogdon JG, Wheeler SB, Samuel CA. The Affordable Care Act and Ethnic Disparities in Colorectal Cancer Screening. American journal of preventive medicine. 2020;58(2):175-81.

18. Mehta SJ, Polsky D, Zhu J, Lewis JD, Kolstad JT, Loewenstein G, et al. ACA-mandated elimination of cost sharing for preventive screening has had limited early impact. The American journal of managed care. 2015;21(7):511-7.

19. Moss HA, Havrilesky LJ, Chino J. Insurance coverage among women diagnosed with a gynecologic malignancy before and after implementation of the Affordable Care Act. Gynecologic oncology. 2017;146(3):457-64.

20. Steenland M, Sinaiko A, Glynn A, Fitzgerald T, Cohen J. The effect of the Affordable Care Act on patient out-of-pocket cost and use of preventive cancer screenings in Massachusetts. Preventive medicine reports. 2019;15:100924.

21. Xiao D, Zheng C, Jindal M, Johnson LB, DeLeire T, Shara N, et al. Medicaid Expansion and Disparity Reduction in Surgical Cancer Care at High-Quality Hospitals. Journal of the American College of Surgeons. 2018;226(1):22-9.

\section{Figures}

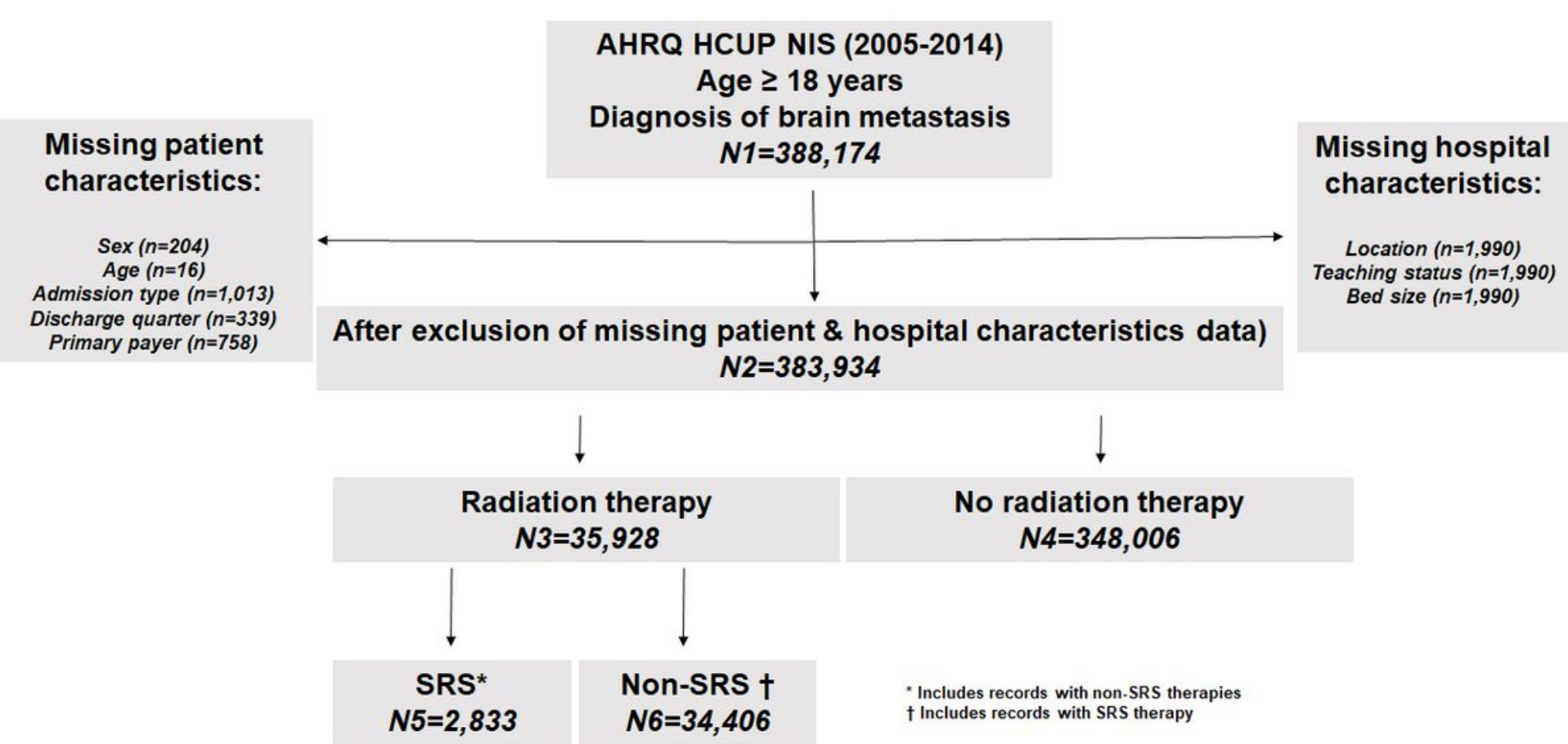

Page $12 / 14$ 
Figure 1

Study flowchart - Nationwide Inpatient Sample (2005-2014)

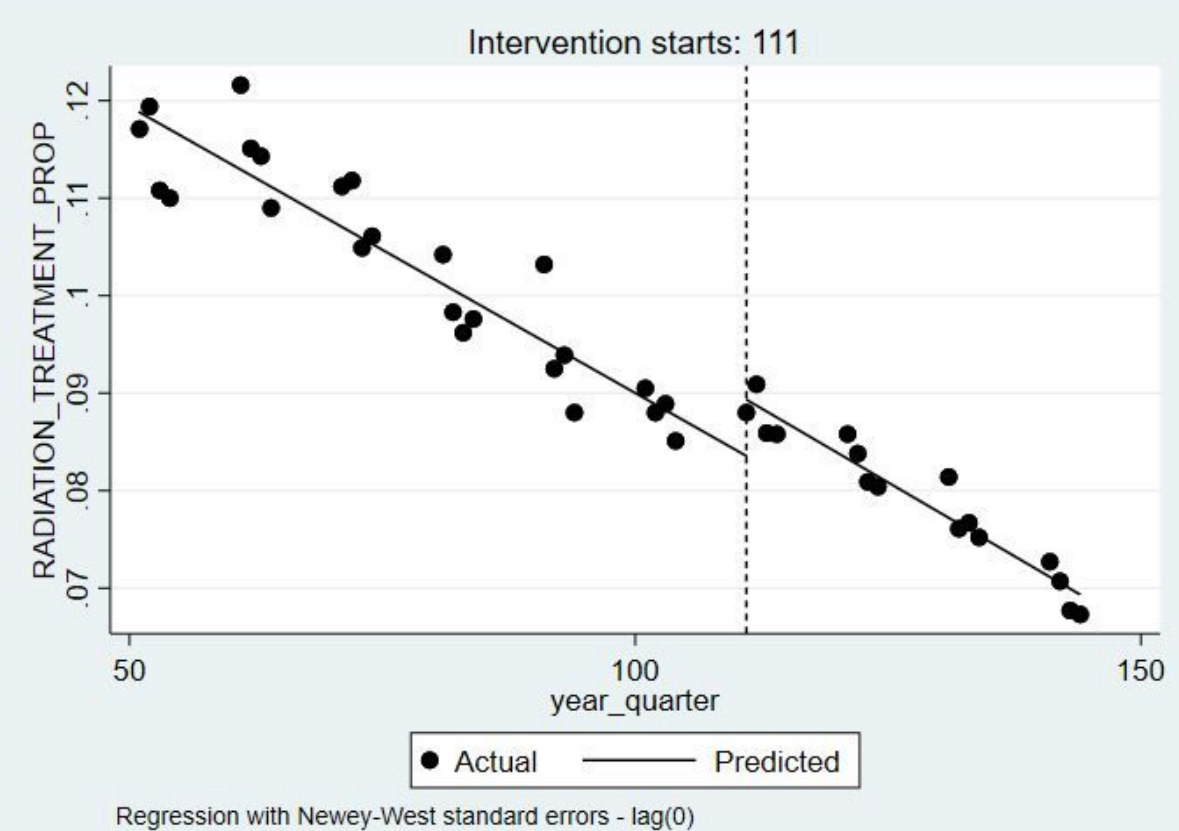

Figure 2

Interrupted time-series analyses for prevalence of radiation therapies - Nationwide Inpatient Sample (2005-2014) Note: 111 represents the 1st quarter of the year 2011.

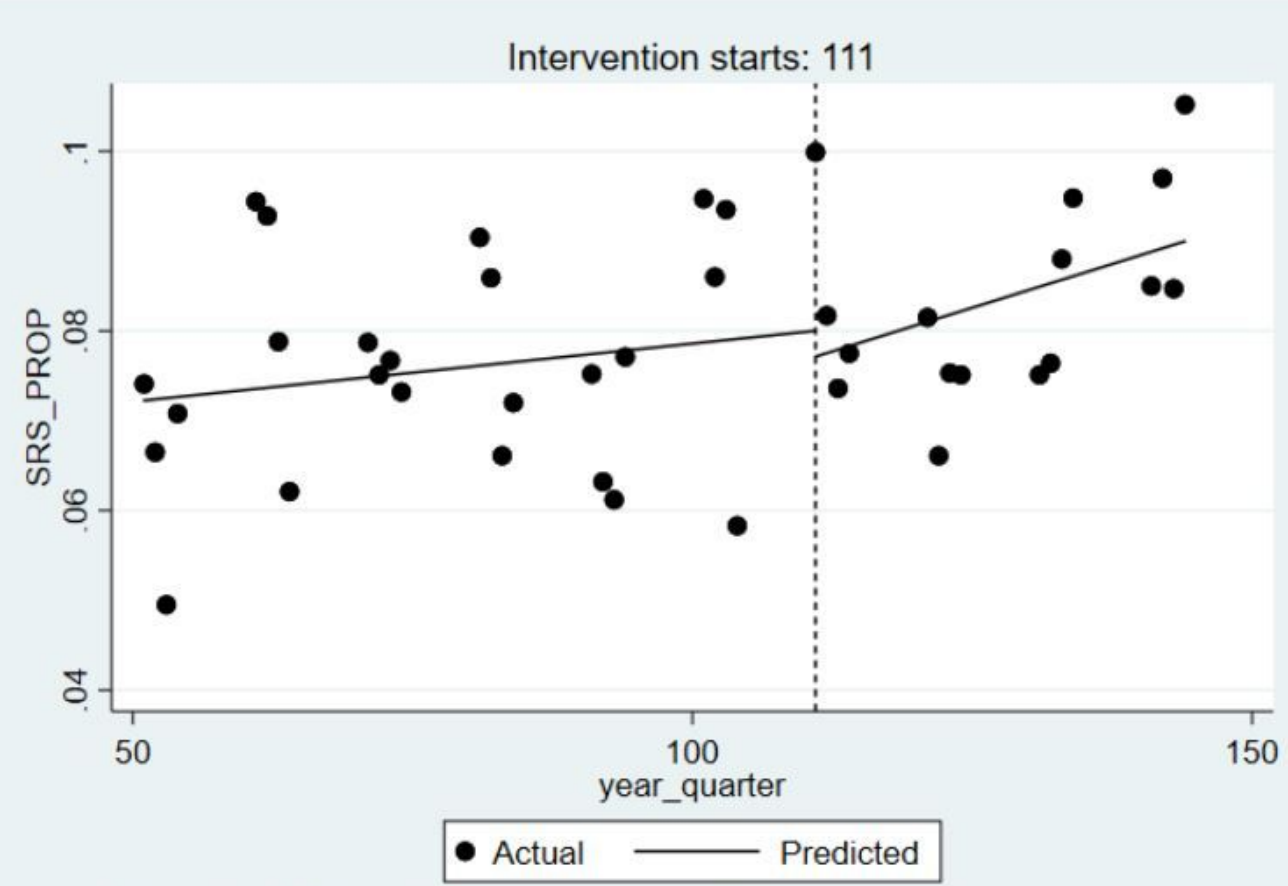

Regression with Newey-West standard errors - $\operatorname{lag}(0)$

Figure 3 
Interrupted time-series analyses for prevalence of stereotactic radiosurgery - Nationwide Inpatient Sample (2005-2014) Note: 111 represents the 1st quarter of the year 2011 .

Page $14 / 14$ 